\title{
Time delays from one-photon transitions in the continuum
}

\section{Journal Article}

\section{Author(s):}

Fuchs, Jaco; Douguet, Nicolas; Donsa, Stefan; Martin, Fernando; Burgdörfer, Joachim; Argenti, Luca; Cattaneo, Laura; Keller, Ursula (i)

Publication date:

2020-02-20

\section{Permanent link:}

https://doi.org/10.3929/ethz-b-000402038

\section{Rights / license:}

Creative Commons Attribution 4.0 International

Originally published in:

Optica 7(2), https://doi.org/10.1364/OPTICA.378639 


\title{
Time delays from one-photon transitions in the continuum
}

\author{
Jaco Fuchs, ${ }^{1, *}$ (i) Nicolas Douguet, ${ }^{2}$ Stefan Donsa, ${ }^{3}$ Fernando Martin, ${ }^{4,5,6}$ \\ Joachim Burgdörfer, ${ }^{3}$ Luca Argenti, ${ }^{2,7}$ Laura Cattaneo,,${ }^{1}$ and Ursula Keller ${ }^{1}$ (1) \\ ${ }^{1}$ Department of Physics, Eidgenössische Technische Hochschule Zürich, Zürich, Switzerland \\ ${ }^{2}$ Department of Physics, University of Central Florida, Orlando, Florida 32186, USA \\ ${ }^{3}$ Institute of Theoretical Physics, Vienna University of Technology, Vienna, Austria \\ ${ }^{4}$ Departamento de Química Modulo 13, Universidad Autónoma de Madrid, 28049 Madrid, Spain \\ ${ }^{5}$ Condensed Matter Physics Center (IFIMAC), Universidad Autonoma de Madrid, 28049 Madrid, Spain \\ ${ }^{6}$ Instituto Madrileño de Estudios Avanzados en Nanociencia (IMDEA-Nano), 28049 Madrid, Spain \\ ${ }^{7}$ CREOL, University of Central Florida, Orlando, Florida 32186, USA \\ ${ }^{*}$ Corresponding author: jafuchs@phys.ethz.ch
}

Received 23 September 2019; revised 22 December 2019; accepted 24 December 2019 (Doc. ID 378639); published 3 February 2020

\begin{abstract}
Attosecond photoionization time delays reveal information about the potential energy landscape that an outgoing electron wavepacket probes upon ionization. In this study, we experimentally quantify the dependence of the time delay on the angular momentum of the liberated photoelectrons. For this purpose, we resolved electron quantum-path interference spectra in energy and angle using a two-color attosecond pump-probe photoionization experiment in helium. A fitting procedure of the angle-dependent interference pattern allows us to disentangle the relative phase of all four quantum pathways that are known to contribute to the final photoelectron signal. In particular, we resolve the dependence on angular momentum of the delay of one-photon transitions between continuum states, which is an essential and universal contribution to the total photoionization delay observed in attosecond pump-probe measurements. For such continuum-continuum transitions, we measure a delay between outgoing $s$ and $d$ electrons as large as 12 attoseconds, close to the ionization threshold in helium. Both single-active-electron and first-principles ab initio simulations confirm this observation for helium and hydrogen, demonstrating the universality of the observed delays.
\end{abstract}

Published by The Optical Society under the terms of the Creative Commons Attribution 4.0 License. Further distribution of this work must maintain attribution to the author(s) and the published article's title, journal citation, and DOI.

https://doi.org/10.1364/OPTICA.378639

\section{INTRODUCTION}

Free electrons cannot exchange photons with a light pulse. Unbound electrons, however, which are subject to an external potential, can absorb (inverse Bremsstrahlung) or emit (stimulated Bremsstrahlung) quanta of the radiation field. In the presence of an attractive Coulomb potential of a nearby ion, the absorption and emission of a single photon promote dipole transitions that change the quantum state. These transitions involve bound as well as continuum states, giving rise to various types of radiative processes such as excitation (bound to bound), ionization [bound to continuum (bc)], recombination (continuum to bound), and continuum-continuum (cc) transitions.

Recent progress in attosecond science has given direct access to timing information in photon-atom interaction on the attosecond scale. In particular, single-photon ionization and the corresponding Eisenbud-Wigner-Smith (EWS) delay [1-5] have attracted much attention. Briefly, due to propagation across the potentialenergy landscape, the excited photoelectron wave packet acquires an energy-dependent phase that results in a measurable group delay, referred to as a photoionization time delay. Relative delays between wave packets from different species [6-8], ionization channels [9-15], and emission angles [16,17] have been measured to very high accuracy and serve as benchmarks for time-dependent quantum mechanical simulations in atoms $[6,8,10,12,16,17]$, molecules $[7,11,14,15]$, and solids $[9,18]$. To date, these attosecond measurement techniques have been based on the delay between two coherent laser pulses that are typically in the extreme ultraviolet (XUV) and the infrared (IR). Thus, the time delays could be measured only between ionization pathways involving at least two photons. In particular, if the first photoabsorption event is a bc transition, a second transition in the continuum is required to access temporal information in state-of-the-art experiments. Thus, in addition to the EWS delay [1,2], the experimentally observed delays contain two more contributions. The first one originates from the spectral phase of the ionizing attosecond pulse train (APT) $[19,20]$ and cancels out when comparing different species or channels. The second contribution originates from the cc transitions mediated by the probing IR laser pulse [3,5,21]. Although it is well known $[4,22,23]$ that the cc contribution to 
the photoionization time delay can be comparable to or even larger than the EWS delay for single photon ionization, it has not drawn much attention until recently. Moreover, since experimentally disentangling the contributions has not been possible so far, time delays of one-photon ionization were accessible only when referencing theoretical calculations $[3,13,22]$.

Recently, experimental evidence of a strong effect of IR-induced cc transitions on the angular dependence of the total photoemission delays has been reported [16,17], stimulating several independent investigations on the origin of this effect [23-29]. In this work, we present a new method that allows us to unravel the delay between electron wave packets from different one-photon transitions in the continuum, purely from experimental data, and independently of the Wigner and XUV contributions. We obtain for the first time access to the angular momentum dependence of the EWS delay for cc transitions. The method is based on an algorithm developed to analyze angularly resolved reconstruction of attosecond beating by interference of two-photon transitions [30] (RABBITT) spectra. We find a ubiquitous positive and energy-dependent time delay, as large as 12 between $s$ and $d$ wave photoelectrons produced by the additional IR-photon exchange that follows photoionization of atomic helium by an XUV ATP. This result is the first demonstration of a direct measurement of the EWS delay arising from one-photon transitions within the continuum. Using two independent computational methods to solve the time-dependent Schrödinger equation (TDSE), one based on the single-active-electron approximation and the other being a first-principles ab initio approach, we obtain excellent agreement with the experimentally retrieved ionization time delays. These findings confirm that, in helium, at energies close to the first ionization threshold, the delay associated with radiative transitions in the continuum is dominated by the electron angular momentum and radial momentum distribution, whereas electronic correlation plays no significant role.

The following section develops the theoretical framework needed to interpret the experiment and the simulations. The experimental and theoretical analyses are described in Sections 3 and 4, respectively. The main results are examined in Section 5, and in the last section, we offer our conclusions.

\section{THEORETICAL FRAMEWORK}

In the photoionization of helium from its $1 s^{2}$ ground state by absorption of a single XUV photon, the total angular momentum of the combined atom-photon system has to be conserved. In the energy range examined in this work, the residual $\mathrm{He}^{+}$ion remains in its lowest ${ }^{2} S(1 s)$ state. The photon angular momentum, therefore, is transferred entirely to the ejected electron, which is emitted as a $p$ wave (angular momentum $l=1$ ). This liberated electron can subsequently absorb (or emit) an additional IR photon through a cc transition, which transfers to the photoelectron additional angular momentum resulting in either an $s$ wave $(l=0)$ or $d$ wave $(l=2)$. For collinear parallelly polarized XUV and IR pulses, as those employed in this study, the magnetic quantum number remains zero.

In the RABBITT technique [30,31], an XUV pulse train consisting of odd harmonics of the fundamental IR laser frequency $\omega$ is used to ionize the target, leading to single-photon peaks (mainbands) in the photoelectron spectrum separated by twice the laser photon energy $2 \hbar \omega$. A weak replica of the fundamental IR with frequency $\omega$ then triggers $\mathrm{cc}$ transitions from the mainband to sidebands with kinetic energies lying between the mainbands. For ionization from an $s$ shell, four main quantum pathways contribute to each sideband, namely, the transitions $s \rightarrow p \rightarrow s$ and $s \rightarrow p \rightarrow d$, for both the absorption and stimulated emission of the IR photon, as illustrated schematically in Fig. 1a. At low IR intensities (less than a few $\mathrm{GW} / \mathrm{cm}^{2}$ at $800 \mathrm{~nm}$ ), pathways to the sidebands that involve the exchange of more than one IR photon give a negligible contribution, and hence, states with angular momentum higher than two are not populated. Due to the interference of absorption and emission pathways, the sideband signal oscillates as a function of the delay $\tau$ between the IR pulse and the XUV pulse train. In Fig. 1b, we show the spectrum of the XUV pulse train and the corresponding photoelectron energy spectrum (PES) of one-photon and two-photon pathways.

In the weak-field regime, the $s(l=0)$ and $d(l=2)$ photoionization amplitudes at the sideband with energy $E_{f}$ can be expressed,
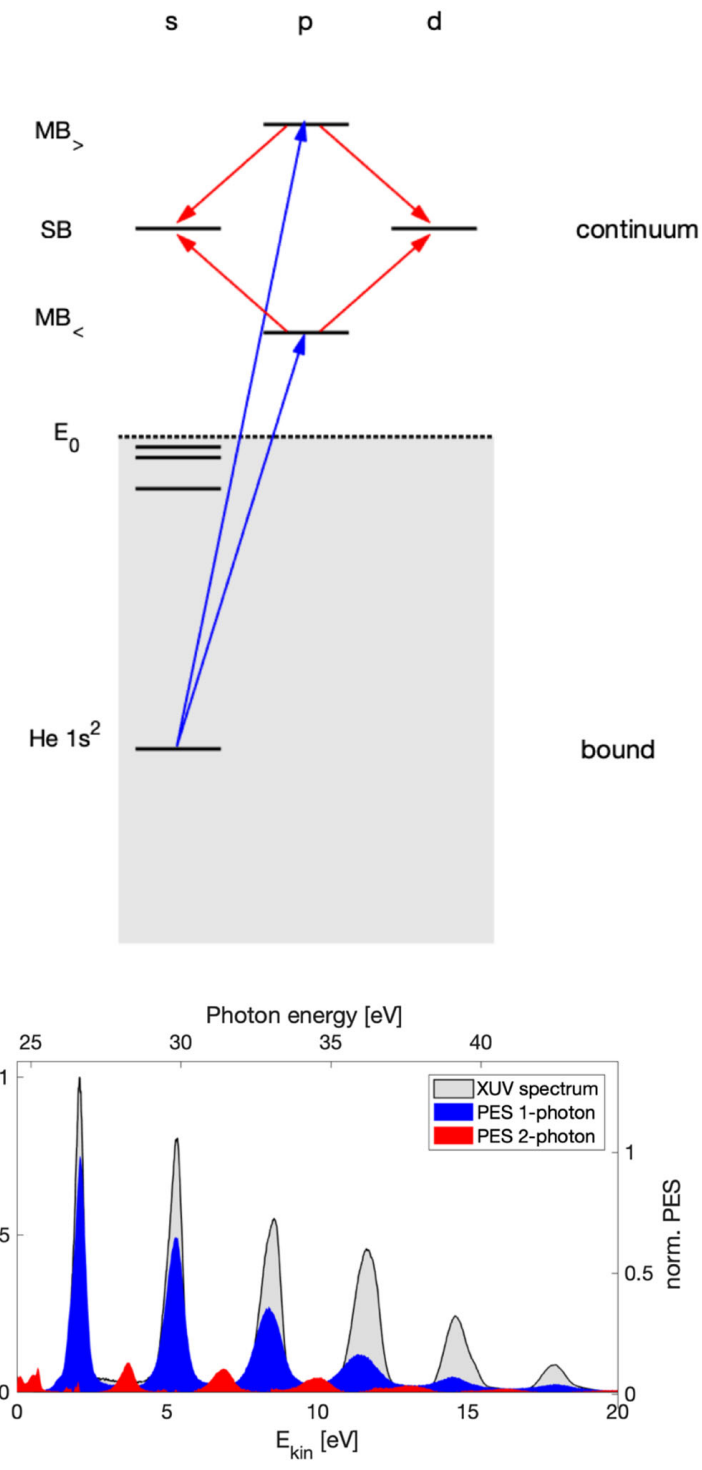

Fig. 1. (a) Quantum pathways leading to the same sideband. The XUV (blue) mediates bound to continuum transitions (ionization); the IR (red) mediates transitions within the continuum. All quantum pathways interfere. (b) Experimental XUV and photoelectron energy spectra (PES). The one-photon PES is obtained by an XUV-only measurement. The two-photon PES is obtained by subtracting the one-photon spectrum from the time-integrated RABBITT spectrum. 
within lowest order of perturbation theory, by the well-known twophoton-transition formula [32]

$$
\begin{aligned}
A_{l}^{(2)}\left(E_{f}\right) & =-i \int \mathrm{d} \Omega M_{E_{f}, l}^{(2)}(\Omega) E_{\mathrm{XUV}}(\Omega) E_{\mathrm{IR}}\left(\left[E_{f}-I_{p}\right] / \hbar-\Omega\right) \\
& =A_{l}^{(2+)}+A_{l}^{(2-)},
\end{aligned}
$$

where $M_{E_{f}, l}^{(2)}$ is the two-photon matrix element, $I_{p}$ is the ionization potential, and $E_{\mathrm{XUV}}$ and $E_{\mathrm{IR}}$ are the Fourier transforms of the XUV and IR electric field, respectively.

The frequency (energy) integral in Eq. (1) can be split into an interval with $0<\Omega<\left(E_{f}-I_{p}\right) / \hbar, A_{l}^{(2+)}$, which corresponds to pathways with absorption of an IR photon and an integral with $\Omega \geq\left(E_{f}-I_{p}\right) / \hbar, A_{l}^{(2-)}$, for the pathways with stimulated emission of an IR photon. Following [21,22], for a narrowband IR spectrum with frequency $\omega$ far from resonances, the phase of the two-photon matrix element [Eq. (1)] can be decomposed into three additive contributions:

$$
A_{l}^{(2 \pm)}=\left|A_{l}^{(2 \pm)}\right| e^{i\left(\varphi_{l}^{c c \pm}+\varphi^{b c} \pm \omega \tau\right)},
$$

with $\varphi_{l}^{\text {cct }}$ the phase of the cc transition with final angular momentum $l, \varphi_{\lessgtr}^{\mathrm{bc}}$ the phase of the one-photon bc transition to the lower $(<)$ or upper $(>)$ mainband, and the phase $\pm \omega \tau$ due to the pumpprobe delay $\tau$, which leads to oscillations in the interference pattern. The resulting ionization probability at the sideband is

$$
\begin{aligned}
I(\vartheta, \varphi, \tau)= & \mid\left(A_{s}^{(2+)}+A_{s}^{(2-)}\right) Y_{0}^{0}(\vartheta, \varphi) \\
& +\left.\left(A_{d}^{(2+)}+A_{d}^{(2-)}\right) Y_{2}^{0}(\vartheta, \varphi)\right|^{2} \\
= & \sum_{n=0}^{4} \beta_{n}(\tau) P_{n}[\cos (\theta)],
\end{aligned}
$$

where $\theta$ is the angle between the common laser polarization axis of the XUV and IR electric field, and the direction of the outgoing electron. The series expansion in Legendre polynomials $P_{n}$ extends up to fourth order [33]. The coefficients $\beta_{n}(\tau)$, which quantify the photoemission anisotropy, have the following expressions:

$$
\begin{aligned}
\beta_{0}= & \left|\mathcal{A}_{s}^{(2+)}\right|^{2}+\left|\mathcal{A}_{s}^{(2-)}\right|^{2}+\left|\mathcal{A}_{d}^{(2+)}\right|^{2}+\left|\mathcal{A}_{d}^{(2-)}\right|^{2} \\
& +2\left|\mathcal{A}_{s}^{(2+)}\right|\left|\mathcal{A}_{s}^{(2-)}\right| \cos \left(2 \omega \tau+\varphi_{s}^{(2+)}-\varphi_{s}^{(2-)}\right) \\
& +2\left|\mathcal{A}_{d}^{(2+)}\right|\left|\mathcal{A}_{d}^{(2-)}\right| \cos \left(2 \omega \tau+\varphi_{d}^{(2+)}-\varphi_{d}^{(2-)}\right), \\
\beta_{2}= & \frac{10}{7}\left[\left|\mathcal{A}_{d}^{(2+)}\right|^{2}+\left|\mathcal{A}_{d}^{(2-)}\right|^{2}\right. \\
& \left.+2\left|\mathcal{A}_{d}^{(2+)}\right|\left|\mathcal{A}_{d}^{(2-)}\right| \cos \left(2 \omega_{\tau}+\varphi_{d}^{(2+)}-\varphi_{d}^{(2-)}\right)\right] \\
& +2 \sqrt{5}\left[\left|\mathcal{A}_{s}^{(2+)}\right|\left|\mathcal{A}_{d}^{(2+)}\right| \cos \left(\varphi_{s}^{(2+)}-\varphi_{d}^{(2+)}\right)\right. \\
& +\left|\mathcal{A}_{s}^{(2-)}\right|\left|\mathcal{A}_{d}^{(2-)}\right| \cos \left(\varphi_{s}^{(2-)}-\varphi_{d}^{(2-)}\right) \\
& +\left|\mathcal{A}_{s}^{(2+)}\right|\left|\mathcal{A}_{d}^{(2-)}\right| \cos \left(2 \omega \tau+\varphi_{s}^{(2+)}-\varphi_{d}^{(2-)}\right) \\
& \left.+\left|\mathcal{A}_{s}^{(2-)}\right|\left|\mathcal{A}_{d}^{(2+)}\right| \cos \left(2 \omega \tau+\varphi_{d}^{(2+)}-\varphi_{s}^{(2-)}\right)\right],
\end{aligned}
$$

$$
\begin{aligned}
\beta_{4}= & \frac{18}{7}\left[\left|\mathcal{A}_{d}^{(2+)}\right|^{2}+\left|\mathcal{A}_{d}^{(2-)}\right|^{2}\right. \\
& \left.+2\left|\mathcal{A}_{d}^{(2+)}\right|\left|\mathcal{A}_{d}^{(2-)}\right| \cos \left(2 \omega \tau+\varphi_{d}^{(2+)}-\varphi_{d}^{(2-)}\right)\right] .
\end{aligned}
$$

Since $s$ and $d$ waves have the same (even) parity, the odd anisotropy parameters $\beta_{1}$ and $\beta_{3}$ are identically zero. Here,

$$
\varphi_{s, d}^{(2 \pm)}=\varphi_{s, d}^{\mathrm{cc} \pm}+\varphi_{\lessgtr}^{\mathrm{bc}}
$$

contains both the phase $\varphi_{s, d}^{\mathrm{cc} \pm}$ of the cc transition and the phase $\varphi_{\lessgtr}^{\mathrm{bc}}$ associated with the preceding ionization. The latter one contains the phase of the ionizing XUV pulse and the atomic phase $\delta_{l}^{\mathrm{bc}}$ for the half-scattering process of the outgoing electron wave packet at the atomic potential. Its spectral derivative $d \delta_{l}^{\mathrm{bc}}(E) / d E$ gives the EWS delay for single-photon ionization. The phase of the cc transition $\varphi_{s, d}^{\mathrm{cc} I}$ then leads to an additional delay often referred to as $\mathrm{cc}$ delay [21] or Coulomb laser coupling (CLC) delay [5]. However, since the IR-driven cc transition occurs primarily at large distances from the atomic core [22], the accumulated phase, unlike for one-photon ionization, does not account for the full half-scattering phase but only for propagation in the long-range tail of the atomic potential. Therefore, the influence of the centrifugal potential $L^{2} / 2 r^{2}$ on the cc phase was previously neglected in the analytical approximation to $\phi^{\mathrm{cc}}$ [22].

\section{EXPERIMENTAL RESULTS}

In order to measure the time- and angle-dependent ionization probability [Eq. (3)], we use a COLTRIMS detector [34] in combination with an XUV-IR pump-probe setup. An amplified Ti-sapphire laser, with a repetition rate of $10 \mathrm{kHz}$, generates a $790 \mathrm{~nm}$ IR pulse of $29 \mathrm{fs}$ FWHM duration and $0.7 \mathrm{~mJ}$ total energy. The pulse is split into an intense (80\%) and a weaker (20\%) component. The stronger IR beam is focused into an argon gas cell where the XUV harmonics of 13-25, corresponding to an energy range of 20 to $40 \mathrm{eV}$, are created by high-harmonic generation [35] (see Fig. 1b). The remaining IR pulse passes through a delay stage and is recombined with the XUV beam, before being focused into a cold helium jet. With the COLTRIMS detector, we measure the three-dimensional momentum of both photoelectrons and ions with a $4 \pi$ solid angle detection capability. With coincidence selection (time-of-flight filtering of the helium ion and momentum conservation condition), we can discriminate against electrons from other reactions. The delay between the two pulses is controlled via a piezo-driven delay stage in combination with active interferometric stabilization. For details on the experimental setup, the reader is referred to [36].

To guarantee a uniform detection capability over all emission angles and energies, we calibrate the detector efficiency using a helium XUV-only measurement, where the differential cross section is known accurately. For the time-resolved measurements, an IR intensity of $3 \cdot 10^{11} \mathrm{~W} / \mathrm{cm}^{2}$ is used at the interaction region. The angle- and time-dependent photoelectron spectra are recorded for 40 delay steps. The resulting angular distributions are then projected on the Legendre polynomials [Eq. (3)] to retrieve the anisotropy parameters of the distribution, which are shown in Fig. 2. The sideband signal is integrated over $0.5 \mathrm{eV}$, as indicated for sideband 18 by the yellow lines.

Figure 3 shows the anisotropy parameters for sideband 18 as a function of the time delay. Each of the three beta parameters 

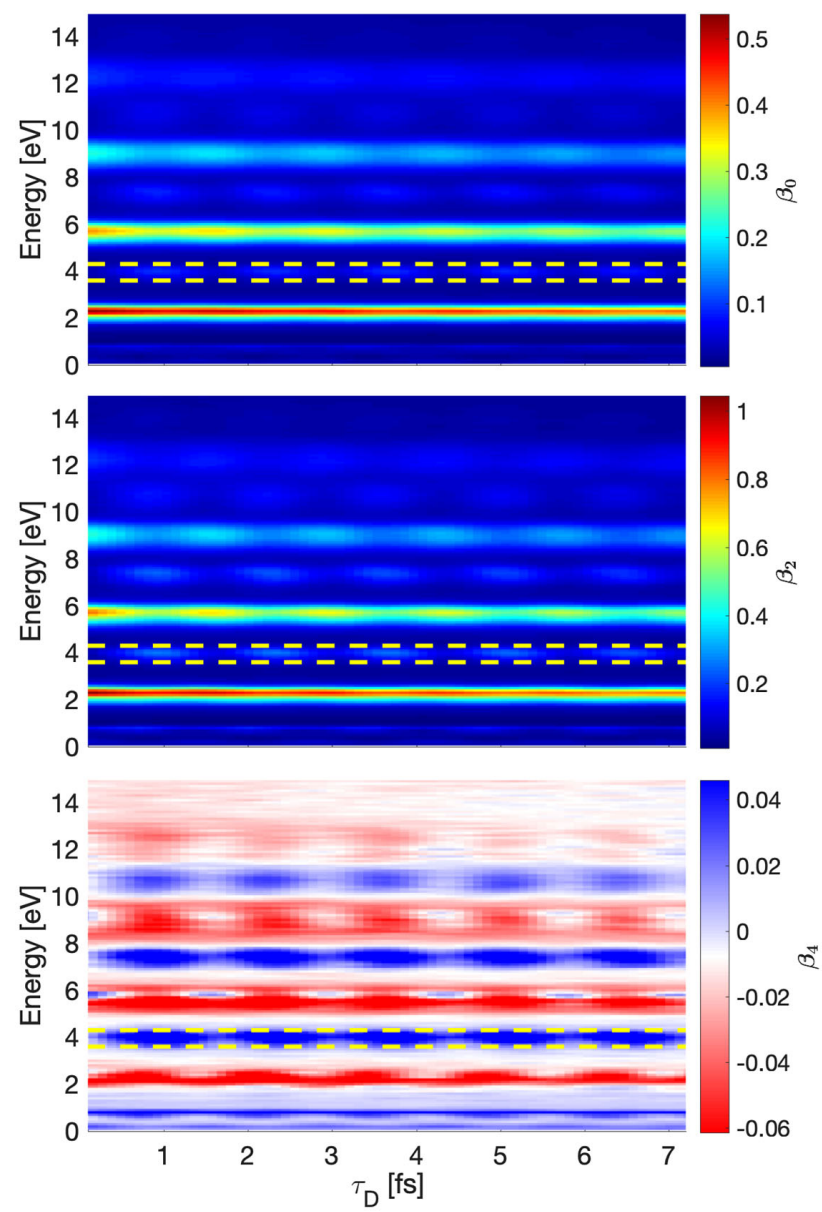

Fig. 2. Experimental anisotropy parameters of the time-resolved photoelectron angular distribution, $\beta_{0}$ (top), $\beta_{2}$ (center), and $\beta_{4}$ (bottom). The yellow lines indicate the integration range of sideband 18 . A positive delay indicates that the IR pulse is delayed with respect to the XUV; the zero delay is chosen arbitrarily.

oscillates at twice the IR frequency, $\beta_{n}=a+b \cos (2 \omega \tau-\varphi)$, with offset $a$, amplitude $b$, and phase $\varphi$, which are related directly to the parameters in Eqs. (4)-(6).

The system of Eqs. (4)-(6) has in total four unknown amplitudes and four unknown phases. The phases appear in differences only and are thus determined only up to an overall constant, allowing us to set one of the phases to zero without loss of generality. The remaining seven variables can then be fitted simultaneously to the system of equations using a least square minimization routine based on the Levenberg-Marquardt algorithm [37]. The convergence of the fit to the correct set of parameters has been tested by performing the same fitting procedure on sets of artificial data, generated directly from Eqs. (4)-(6), both with and without typical measurement noise (up to 5\% relative noise of each anisotropy parameter). For the experimental sampling frequency and number of points, the error of the retrieved phases stays below $0.01 \mathrm{rad}$. Figure 3 shows the fit to the experimental data for sideband 18 . Making use of both the angle-dependent phase and amplitude of the RABBITT interference pattern, we can thus determine the amplitudes and relative phases of all four quantum paths contributing to any given sideband. In particular, the relative phase between the two pathways that lead to different angular momenta is for the absorption,
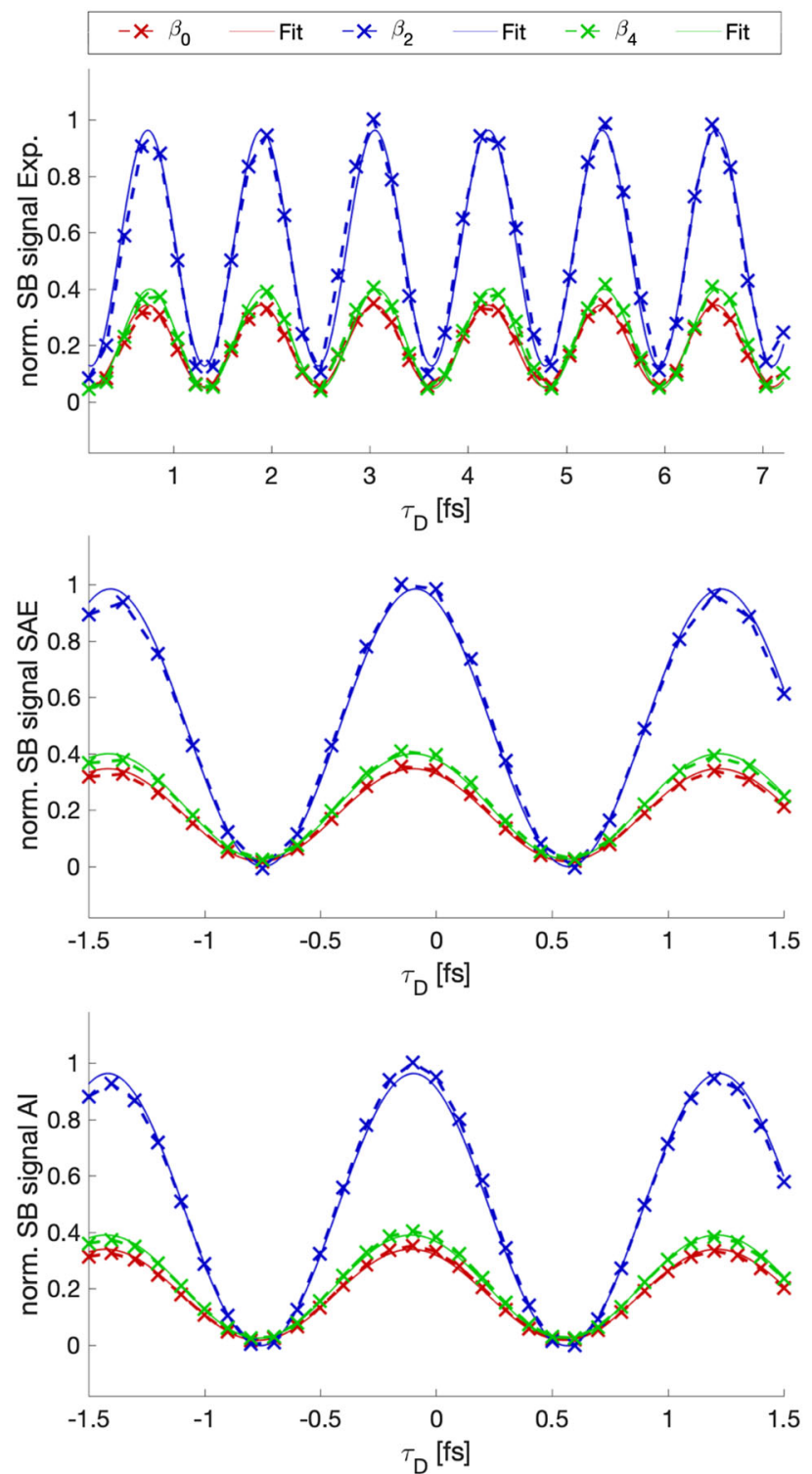

Fig. 3. Simultaneous fit of the sideband anisotropy parameters. The time-resolved sideband signal and the simultaneous fit of the anisotropy parameters $\beta_{0}, \beta_{2}$, and $\beta_{4}$ are shown for sideband 18 for the experimental data (top), the SAE calculations (middle), and the full ab initio (bottom), respectively.

$$
\begin{aligned}
\varphi_{s}^{(2+)}-\varphi_{d}^{(2+)} & =\varphi_{s}^{\mathrm{cc}+}+\varphi_{(<)}^{\mathrm{bc}}-\varphi_{d}^{\mathrm{cc}+}-\varphi_{(<)}^{\mathrm{bc}} \\
& =\varphi_{s}^{\mathrm{cc}+}-\varphi_{d}^{\mathrm{cc}+},
\end{aligned}
$$

and stimulated emission of an IR photon,

$$
\begin{aligned}
\varphi_{s}^{(2-)}-\varphi_{d}^{(2-)} & =\varphi_{s}^{\mathrm{cc}-}+\varphi_{(>)}^{\mathrm{bc}}-\varphi_{d}^{\mathrm{cc}-}-\varphi_{(>)}^{\mathrm{bc}} \\
& =\varphi_{s}^{\mathrm{cc}-}-\varphi_{d}^{\mathrm{cc}-} .
\end{aligned}
$$

This enables to measure directly the influence of the final-state angular momentum on the cc phase independent of the preceding one-photon bound-free transition.

Indeed, in each case, we retrieve the phase difference between pathways involving the same intermediate state, i.e., cc transitions following the absorption of XUV photons of the same energy. Consequently, the phase of the bc transition, which includes both 


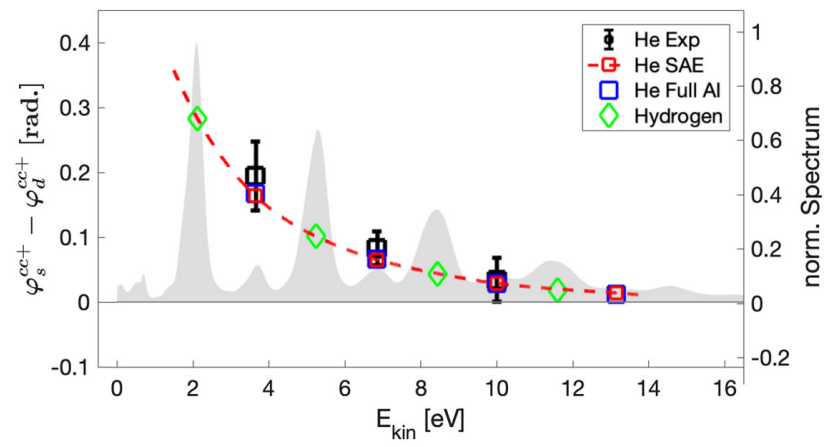

Fig. 4. Difference $\varphi_{s}^{\mathrm{cc}+}-\varphi_{d}^{\mathrm{cc}+}$ in radians for the $\mathrm{cc}$ transition involving absorption. The experimental values represent the mean values of four measurements, and the error bars correspond to the uncertainty of the mean. The discrepancy between the two simulations lies below $2 \%$ of their absolute value. The shaded area represents the PES given in Fig. 1.

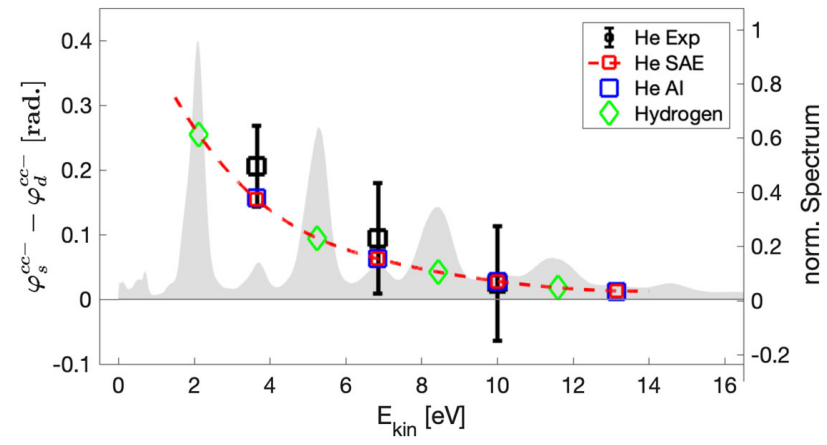

Fig. 5. Difference $\varphi_{s}^{\mathrm{cc}-}-\varphi_{d}^{\mathrm{cc}-}$ for the cc transition involving stimulated emission; otherwise, same as Figure 4.

XUV chirp and the $p$-wave scattering phase, cancels out, such that the remaining phase difference is due purely to the one-photon transition in the continuum.

In contrast to the traditional RABBITT analysis $[19,20]$, where phase differences $\varphi(E+\omega)-\varphi(E-\omega)$ are extracted in order to approximate the phase derivative, our method yields an (absolute) phase difference at a fixed energy. In detail, in RABBITT, the total angle-integrated sideband phase contains the phase difference between pathways originating from neighboring harmonics, i.e., an approximated phase derivative across two harmonics. Therefore, even when comparing different species, the measured delays correspond to differences in derivatives, or respectively, differences in group delay. As a consequence, absolute phase differences remain hidden. In contrast, by comparing pathways following the absorption of the same harmonic, the present procedure allows us to extract an absolute phase difference between two pathways.

Figures 4 and 5 show the mean of the experimentally retrieved phases for sidebands 18,20 , and 22 , averaging over four independent measurements. The error bars represent the uncertainty of the mean, indicating an estimate for the total uncertainty of the combined measurement and fitting procedure.

\section{THEORETICAL RESULTS}

In order to prove the validity of the present extraction method, we apply the same fitting procedure to computed RABBITT traces, for which the total phase of the two-photon electron wave packet and thus the cc phase can be accessed directly. We performed single-active-electron (SAE) calculations [38,39], where the TDSE for helium is solved using the potential of Ref. [40] and a finitedifference scheme based on the Crank-Nicolson method. The sideband signal is analyzed in the same way as the experimental data and illustrated with the corresponding fit in Fig. 3 (center). The retrieved values for the phase difference between the $s$ - and $d$-final-state partial waves are shown in Figs. 4 and 5 for sidebands 18-24. In addition, by using a single harmonic in the TDSE calculations, we retrieve directly the phase of the outgoing $s$ and $d$ waves at the neighboring sidebands without the need to invoke interferences of different pathways. Using the latter procedure, we find excellent agreement (below 0.015 rad absolute difference) with the fitted phases, thereby confirming the validity of the present extraction method.

To probe for the possible influence of electron correlation and exclude possible shortcomings of the SAE approximation, we additionally perform full $a b$ initio simulations using the timedependent close-coupling method [41] on a spatial finite element discrete variable representation grid [42], thereby solving the full two-electron TDSE for atomic helium from first principles $[43,44]$. The electric fields are treated in the dipole approximation. Both, the ab initio and the SAE simulation employ an IR pulse with central wavelength of $790 \mathrm{~nm}$ and a Gaussian envelope with $8 \mathrm{fs}$ FWHM. The spectral amplitude and phase of each harmonic were chosen to match the experimental spectrum. We have checked for the potential influence of the IR pulse duration on the extracted phases. The excellent agreement of the results from SAE simulations for two different IR pulse durations of $8 \mathrm{fs}$ and $20 \mathrm{fs}$ (FWHM) allows one to rule out any significant pulse duration effects on the resulting phases.

Comparing the results of the two independent simulation methods with the experiment in Figs. 4 and 5, we observe excellent agreement among all three data sets. We therefore can conclude that the effect of electron correlation on the cc transition is negligible or identical to the contributing pathways in the investigated energy range.

In addition, we report calculations for the hydrogen atom, for which harmonics from the ninth to the 17 th order are used to generate the XUV spectrum, such that the electron kinetic energy remains in the same range as for helium. It can be observed in Figs. 4 and 5 that the retrieved phase delays exactly follow the helium trend, thus supporting the argument of negligible influence from both electron correlation effects and the helium short-range potential on the investigated cc transition time delays.

\section{DISCUSSION}

We observe a remarkable quantitative agreement between the experiment and theoretical values for the phase difference $\varphi_{s}^{\mathrm{cc} \pm}-\varphi_{d}^{\mathrm{cc} \pm}$ for electron energies $2 \mathrm{eV} \leq \mathrm{E} \leq 14 \mathrm{eV}$ obtained from two independent computational methods for both hydrogen and helium. We have found a significant phase difference with a maximum value of $0.21 \mathrm{rad}$ between $s$ and $d$ partial waves at harmonic 18 corresponding to a final state with energy of $3.7 \mathrm{eV}$. Our data reveal three main features:

i. The relative phase between the $s$ and $d$ partial waves is ubiquitously positive and decreases with energy for cc transitions 
at all kinetic energies, whether involving absorption or stimulated emission.

ii. At all sidebands, the absolute values of the phase difference between $s$ and $d$ waves are almost equal for absorption and stimulated emission. The discrepancy lies far below the experimentally accessible precision. The theoretical values indicate slightly larger delays for absorption.

iii. The cc phase difference converges to zero with increasing kinetic energy.

Although the observed phase difference has not been experimentally identified in the past, the above observations were already visible in earlier numerical simulations [23], and they had been predicted by Dahlström and co-workers [21,25,27,45] (see also supplemental material in [17]).

Qualitatively, the trend mirrors the observation of partial-wave interferences, resulting in electron-spin polarization in one-photon bc transitions [46-48]. The observations (i)-(iii) are fully consistent with the influence of the final-state centrifugal potential on the continuum scattering phase and on the EWS time delay, in the present case for cc transitions. Observation (iii) is the obvious consequence of the decreasing effect of the underlying potential energy landscape on the escaping electron. For increasing energies, the wave function tends towards the behavior of a free spherical wave for which all delays vanish. Moreover, with increasing momentum of the outgoing wave packet, the cc transition is effectively shifted to larger distances from the ionic core at which the centrifugal potential $\propto 1 / r^{2}$ becomes negligible compared to the Coulomb potential. The latter was the underpinning of previous analytic estimates of the cc phase and time delay in which the angular momentum dependence was neglected [22].

Observation (i) clearly shows that the cc phase is in fact related directly to the EWS phase for cc scattering. This is supported by the observation that the phase and corresponding delay qualitatively resemble the EWS delay for bc transitions to different angular momentum states [5,46-48]. The fact that the retrieved phases are significantly smaller (by factors 3-4) relative to the scattering phase is due to the fact that, unlike for the bc transition, the cc transition in the two-photon scenario probes the potential landscape not for the full half-scattering but only at large distances where the centrifugal potential is weaker, yet still leads to clearly resolvable effects at low energies.

Observation (ii) then confirms the fact that the relevant phase is accumulated at distances where the Coulomb potential $(1 / r)$ dominates, the centrifugal potential provides a (small) correction, and short-ranged contributions are entirely negligible. Therefore, the observed phases are universal for a given angular momentum, i.e., independent of the atomic species, and slightly larger for absorption than for emission. The latter is in line with the fact that the outgoing wave packet after the bc transition propagates initially slower before absorption, thereby enhancing the influence of the centrifugal potential on the subsequent cc transition. For the same reason, we thus expect the measured phases to depend on the wavelength of the laser field that drives the cc transitions.

The resulting EWS delay between the $s$ and $d$ partial waves, observed experimentally for the first time in a cc transition, allows for a simple, quasi-classical interpretation: due to the different angular momenta, the rotational and radial energy distributions of the $s$ and $d$ wave-packet components are different, and since the rotational energy fraction is larger for the $d$ wave components, the radial expansion is slower. This implies a positive EWS delay $\frac{d}{d E}\left(\delta_{l=2}(E)-\delta_{l=0}(E)\right)$ of the $d$ wave relative to the $s$ wave, consistent with well-known trends for EWS delays in bc transitions. More generally, one expects larger delays for wave-packet components with higher angular momentum quantum numbers. The experimental confirmation of this effect for cc transitions and the quantification of its energy dependence are the main findings of this work. In the present experiment, in which the electron wave packet's center energy is $6.1 \mathrm{eV}$ and the IR wavelength is $790 \mathrm{~nm}$, the $d$ wave is retarded by 12 as relative to the $s$ wave. Higher-order derivatives of the measured phase difference affect the shape of the envelope and lead to differences in chirp between the $s$ and $d$ partial waves. The implication for the wave packet in time can be inferred from the Fourier integral over the spectral components of absorption and emission pathways of the two partial wave packets. As the phase differences between $s$ and $d$ waves are almost equal for absorption and stimulated emission, using $\Delta \varphi_{s d}=\varphi_{s}^{\mathrm{cc}}-\varphi_{d}^{\mathrm{cc}}$, the partial wave functions can be written in time domain as

$$
\phi_{s}(t)=\int A_{s}^{(2)}(\omega) e^{-i \omega t} \mathrm{~d} \omega
$$

and

$$
\phi_{d}(t)=\int c_{d / s}(\omega) A_{s}^{(2)}(\omega) e^{-i \Delta \varphi_{s d}(\omega)} e^{-i \omega t} \mathrm{~d} \omega,
$$

where $\quad \omega=E_{\mathrm{kin}} / \hbar, \quad$ and $\quad c_{d / s}(\omega)=\left|A_{d}^{(2)}(\omega)\right| /\left|A_{s}^{(2)}(\omega)\right|$ is the absolute amplitude ratio. Approximating the phase difference with a first-order Taylor expansion $\Delta \varphi_{s d}(\omega)=$ $\Delta \varphi_{s d}\left(\omega_{0}\right)+\left.\Delta \varphi_{s d}^{\prime}\right|_{\omega_{0}}\left(\omega-\omega_{0}\right)$ around the center (mean) frequency $\omega_{0}$ of the wave packet and assuming a weakly energydependent amplitude ratio $c_{d / s}(\omega) \approx c_{d / s}\left(\omega_{0}\right)$, it follows immediately that

$$
\begin{aligned}
\phi_{d}(t)= & c_{d / s}\left(\omega_{0}\right) e^{i\left[\Delta \varphi_{s d}^{\prime} \mid \omega_{0} \omega_{0}-\Delta \varphi_{s d}\left(\omega_{0}\right)\right]} \\
& \times \int A_{s}^{(2)}(\omega) e^{-i \omega\left(t+\Delta \varphi_{s d}^{\prime} \mid \omega_{0}\right)} \mathrm{d} \omega \\
= & \phi_{s}\left(t+\left.\Delta \varphi_{s d}^{\prime}\right|_{\omega_{0}}\right) c_{d / s}\left(\omega_{0}\right) e^{-i \Delta \phi_{0}},
\end{aligned}
$$

where $\Delta \phi_{0}=\Delta \varphi_{s d}\left(\omega_{0}\right)-\left.\Delta \varphi_{s d}^{\prime}\right|_{\omega_{0}} \omega_{0}$. The assumption of a flat amplitude ratio is valid only for a narrow energy spectrum. An explicit study of the amplitude ratio for various species is given in $[27,49])$.

Hence, from Eq. (12) it follows that, in addition to the group delay $\tau_{s d}=-\left.\Delta \varphi_{s d}^{\prime}\right|_{\omega_{0}}$, an additional absolute phase offset $\Delta \phi_{0}$ is imparted to the $d$ relative to the $s$ partial wave. Both quantities, delay and absolute phase offset, depend strongly on the wave packet's center energy. The additional phase shift corresponds to an advance of the wave packet's absolute phase with respect to the wave-packet envelope. This phase lag between the $s$ and the $d$ waves in the outgoing wave packet implies an interesting analogue to the carrier-envelope phase (CEP) slip. Although the absolute phase has no impact on the "classical" observables of the electron, i.e., spatial localization and momentum, it determines the interference. We hereby report an effect of the CEP imprinted in the photoelectron wave packet by the multiphoton ionization process. It is illustrated together with the group delay $\tau_{s d}$ as a function of the wave packet's center energy for the phases retrieved from the SAE simulations in Fig. 6. As can be inferred in Figs. 4 and 5, the theoretically calculated cc phase difference in hydrogen follows 


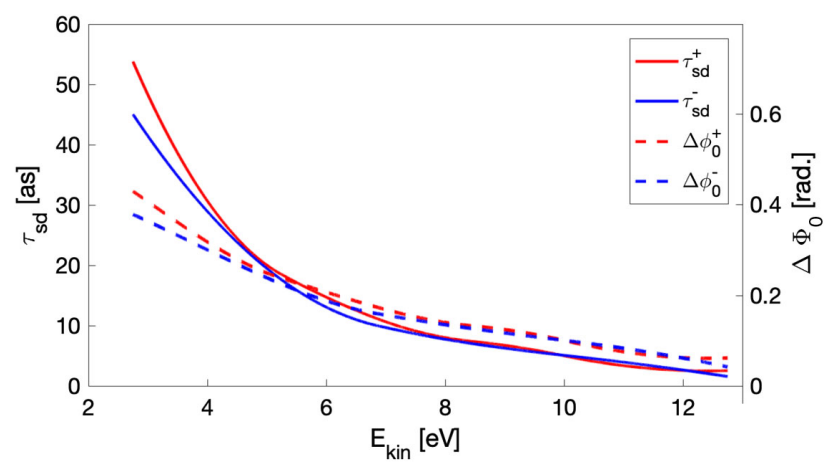

Fig. 6. Delay $\tau_{s d}$ and absolute phase offset $\Delta \phi_{0}$ between the outgoing $d$ and $s$ partial waves as a function of the wave packets' center energy for the phase difference retrieved from the SAE simulations.

the same trend as in helium. This finding further supports the notion that, in systems with small polarizability such as helium, the relative phase between wave-packet components with different angular momenta is a universal feature of laser-assisted Coulomb scattering when the Coulomb and the centrifugal potential dominate any short-range admixtures $[25,45]$. For larger atoms or molecules, where electron correlation effects are more prominent, however, deviations from the observed trend cannot be excluded.

At lower kinetic energies, even larger delays are to be expected. These were not measured in this work due to the limited tunability of the XUV spectrum in the present experiment. Sideband 16, in principle, would lie just above the helium ionization threshold and could be analyzed along those lines. However, higher excited states of the neutral helium atom come into play here [50], noticeable in Fig. 2 (bottom). Including the latter sideband would then involve more complex transitions beyond cc transitions, and is beyond the scope of this work.

\section{CONCLUSION AND OUTLOOK}

In conclusion, we have established an experimental protocol exploiting a novel fitting procedure of angle-resolved RABBITT spectra, which allows us to determine the amplitudes and relative phases of all quantum pathways that contribute to the same final energy in atomic helium. Comparing pathways following the absorption of the same XUV photon, we find a time delay between $s$ and $d$ waves arising from one-photon transitions in the continuum as large as 12 attoseconds. This represents the first measurement of the EWS time delay for (inverse) Bremsstrahlung. Moreover, we find excellent quantitative agreement between the experiment and two independent theoretical simulations. The observed trend reveals ubiquitously positive phase delays between $s$ and $d$ waves for both absorption and stimulated emission. The measured relative phase, which vanishes for high kinetic energies, is determined by the final state of the continuum wavepacket components with different angular momenta populated by the two-photon transition. The radiative transition in the continuum occurs at large distances where the Coulomb potential of the nearby ion and the centrifugal potential dominate the target-dependent short-range potential. As a consequence, the relative phases are expected to be a universal property of radiative transitions in the continuum, which is relevant to characterizing the photoemission dynamics for different atomic species. The same absolute phase difference affects the sideband anisotropy even in the stationary regime, as for example, in the laser-assisted ionization of helium with monochromatic synchrotron radiation.

This work not only serves as a proof-of-principle demonstration for accurately disentangling multiple interfering quantum pathways but also gives new physical insight into the time properties of the fundamental inverse and stimulated Bremsstrahlungs processes. The proposed method can be easily generalized to other systems and cc transitions. The work opens up new experimental opportunities for analyzing and selecting quantum pathways in larger systems such as heavier atoms and molecules, where different quantum pathways can lead to distinct molecular breakup reactions or final states. Additionally, we hope that our study will motivate further experimental and theoretical studies of cc transitions not only in various atomic species, but more generally in small molecules, aiming for a general understanding of intermediate to long-range interactions on the photoemission time delay.

Funding. National Science Foundation (1607588); University of Central Florida (24089045); Vienna Science and Technology Fund (MA14-002); Austrian Science Fund (FWF- W1243Solids4Fun, FWF-SFB041-VICOM, IMPRS-APS); Ministerio de Economía y Competitividad (FIS2016-77889-R, MDM2014-0377, SEV-2016-0686); Schweizerischer Nationalfonds zur Förderung der Wissenschaftlichen Forschung (NCCR MUST).

Disclosures. The authors declare no conflicts of interest.

\section{REFERENCES}

1. E. P. Wigner, "Lower limit for the energy derivative of the scattering phase shift," Phys. Rev. 98, 145-147 (1955).

2. F. T. Smith, "Lifetime matrix in collision theory," Phys. Rev. 118, 349-356 (1960).

3. S. Nagele, R. Pazourek, J. Feist, K. Doblhoff-Dier, C. Lemell, K. Tókési, and J. Burgdörfer, "Time-resolved photoemission by attosecond streaking: extraction of time information," J. Phys. B 44, 081001 (2011).

4. J. M. Dahlström, D. Guénot, K. Klünder, M. Gisselbrecht, J. Mauritsson, A. L'Huillier, A. Maquet, and R. Taïeb, "Theory of attosecond delays in laser-assisted photoionization," Chem. Phys. 414, 53-64 (2013).

5. R. Pazourek, S. Nagele, and J. Burgdörfer, "Attosecond chronoscopy of photoemission," Rev. Mod. Phys. 87, 765-802 (2015).

6. M. Sabbar, S. Heuser, R. Boge, M. Lucchini, T. Carette, E. Lindroth, L. Gallmann, C. Cirelli, and U. Keller, "Resonance effects in photoemission time delays," Phys. Rev. Lett. 115, 133001 (2015).

7. M. Huppert, I. Jordan, D. Baykusheva, A. von Conta, and H. J. Wörner, "Attosecond delays in molecular photoionization," Phys. Rev. Lett. 117, 093001 (2016).

8. M. Sabbar, S. Heuser, R. Boge, M. Lucchini, T. Carette, E. Lindroth, L. Gallmann, C. Cirelli, and U. Keller, "Erratum: resonance effects in photoemission time delays [Phys. Rev. Lett. 115, 133001 (2015)]," Phys. Rev. Lett. 119, 219901 (2017).

9. A. L. Cavalieri, N. Müller, T. Uphues, V. S. Yakovlev, A. Baltuška, B. Horvath, B. Schmidt, L. Blümel, R. Holzwarth, S. Hendel, M. Drescher, U. Kleineberg, P. M. Echenique, R. Kienberger, F. Krausz, and U. Heinzmann, "Attosecond spectroscopy in condensed matter," Nature 449, 1029-1032 (2007).

10. M. Schultze, M. Fiess, N. Karpowicz, J. Gagnon, M. Korbman, M. Hofstetter, S. Neppl, A. L. Cavalieri, Y. Komninos, T. Mercouris, C. A. Nicolaides, R. Pazourek, S. Nagele, J. Feist, J. Burgdörfer, A. M. Azzeer, R. Ernstorfer, R. Kienberger, U. Kleineberg, E. Goulielmakis, F. Krausz, and V. S. Yakovlev, "Delay in photoemission," Science 328, 1658-1662 (2010).

11. G. Sansone, F. Kelkensberg, J. F. Pérez-Torres, F. Morales, M. F. Kling, W. Siu, O. Ghafur, P. Johnsson, M. Swoboda, E. Benedetti, F. Ferrari, F. Lépine, J. L. Sanz-Vicario, S. Zherebtsov, I. Znakovskaya, A. Lhuillier, M. Y. Ivanov, M. Nisoli, F. Martín, and M. J. Vrakking, "Electron localization 
following attosecond molecular photoionization," Nature 465, 763-766 (2010).

12. M. Ossiander, F. Siegrist, V. Shirvanyan, R. Pazourek, A. Sommer, T. Latka, A. Guggenmos, S. Nagele, J. Feist, J. Burgdörfer, R. Kienberger, and M. Schultze, "Attosecond correlation dynamics," Nat. Phys. 13, 280-285 (2017).

13. M. Isinger, R. J. Squibb, D. Busto, S. Zhong, A. Harth, D. Kroon, S. Nandi, C. L. Arnold, M. Miranda, J. M. Dahlström, E. Lindroth, R. Feifel, M. Gisselbrecht, and A. L'Huillier, "Photoionization in the time and frequency domain," Science 358, 893-896 (2017).

14. J. Vos, L. Cattaneo, S. Patchkovskii, T. Zimmermann, C. Cirelli, M. Lucchini, A. Kheifets, A. S. Landsman, and U. Keller, "Orientationdependent stereo Wigner time delay and electron localization in a smal molecule," Science 360, 1326-1330 (2018).

15. L. Cattaneo, J. Vos, R. Y. Bello, A. Palacios, S. Heuser, L. Pedrelli, M. Lucchini, C. Cirelli, F. Martín, and U. Keller, "Attosecond coupled electron and nuclear dynamics in dissociative ionization of $\mathrm{H} 2$," Nat. Phys. 14, 733-739 (2018).

16. S. Heuser, Á. Jiménez Galán, C. Cirelli, C. Marante, M. Sabbar, R. Boge, M. Lucchini, L. Gallmann, I. Ivanov, A. S. Kheifets, J. M. Dahlström, E. Lindroth, L. Argenti, F. Martín, and U. Keller, "Angular dependence of photoemission time delay in helium," Phys. Rev. A 94, 063409 (2016).

17. C. Cirelli, C. Marante, S. Heuser, C. L. Petersson, Á. J. Galán, L. Argenti, S. Zhong, D. Busto, M. Isinger, S. Nandi, S. MacLot, L. Rading, P. Johnsson, M. Gisselbrecht, M. Lucchini, L. Gallmann, J. M. Dahlström, E. Lindroth, A. L'Huillier, F. Martín, and U. Keller, "Anisotropic photoemission time delays close to a Fano resonance," Nat. Commun. 9, 955 (2018).

18. L. Miaja-Avila, C. Lei, M. Aeschlimann, J. L. Gland, M. M. Murnane, H. C Kapteyn, and G. Saathoff, "Laser-assisted photoelectric effect from surfaces," Phys. Rev. Lett. 97, 113604 (2006).

19. Y. Mairesse, D. de Bohan, L. J. Frasinski, H. Merdji, L. C. Dinu, P. Monchicourt, P. Breger, M. Kovacev, R. Taieb, B. Carre, H. G. Muller, P. Agostini, and P. Salieres, "Attosecond synchronization of high-harmonic soft x-rays," Science 302, 1540-1544 (2003).

20. L. Cattaneo, J. Vos, M. Lucchini, L. Gallmann, C. Cirelli, and U. Keller, "Comparison of attosecond streaking and RABBITT," Opt. Express 24 29060 (2016).

21. J. M. Dahlström, A. L'Huillier, and A. Maquet, "Introduction to attosecond delays in photoionization," J. Phys. B 45, 183001 (2012).

22. K. Klünder, J. M. Dahlström, M. Gisselbrecht, T. Fordell, M. Swoboda, D. Guénot, P. Johnsson, J. Caillat, J. Mauritsson, A. Maquet, R. Taïeb, and A. L'Huillier, "Probing single-photon ionization on the attosecond time scale," Phys. Rev. Lett. 106, 143002 (2011).

23. I. A. Ivanov and A. S. Kheifets, "Angle-dependent time delay in two-color XUV+IR photoemission of He and Ne," Phys. Rev. A 96, 13408 (2017).

24. J. Wätzel, A. S. Moskalenko, Y. Pavlyukh, and J. Berakdar, "Angular resolved time delay in photoemission," J. Phys. B 48, 025602 (2015).

25. I. A. Ivanov, J. M. Dahlström, E. Lindroth, and A. S. Kheifets, "On the angular dependence of the photoemission time delay in helium," arXiv. (2016).

26. P. Hockett, "Angle-resolved RABBITT: theory and numerics," J. Phys. B 50, 154002 (2017)

27. D. Busto, J. Vinbladh, S. Zhong, M. Isinger, S. Nandi, S. Maclot, P. Johnsson, M. Gisselbrecht, A. L'Huillier, E. Lindroth, and J. M. Dahlström, "Fano's propensity rule in angle-resolved attosecond pump-probe photoionization," Phys. Rev. Lett. 123, 133201 (2019).

28. A. W. Bray, F. Naseem, and A. S. Kheifets, "Simulation of angularresolved RABBITT measurements in noble-gas atoms," Phys. Rev. A 97, 63404 (2018).

29. X. Zhao, H. Wei, W.-W. Yu, and C. D. Lin, "Reconstruction of the complex angle-dependent photoionization transition dipole from a laser-dressed streaking experiment," Phys. Rev. A 98, 053404 (2018).

30. P. M. Paul, E. S. Toma, P. Breger, G. Mullot, F. Auge, P. Balcou, H. G. Muller, and P. Agostini, "Observation of a train of attosecond pulses from high harmonic generation," Science 292, 1689-1692 (2001).
31. H. Muller, "Reconstruction of attosecond harmonic beating by interference of two-photon transitions," Appl. Phys. B 74, s17-s21 (2002).

32. Á. Jiménez-Galán, F. Martín, and L. Argenti, "Two-photon finite-pulse model for resonant transitions in attosecond experiments," Phys. Rev. A 93, 023429 (2016)

33. G. Laurent, W. Cao, H. Li, Z. Wang, I. Ben-Itzhak, and C. L. Cocke, "Attosecond control of orbital parity mix interferences and the relative phase of even and odd harmonics in an attosecond pulse train," Phys. Rev. Lett. 109, 083001 (2012).

34. R. Dörner, V. Mergel, O. Jagutzki, L. Spielberger, J. Ullrich, R. Moshammer, and H. Schmidt-Böcking, "Cold target recoil ion momentum spectroscopy: a 'momentum microscope' to view atomic collision dynamics," Phys. Rep. 330, 95-192 (2000).

35. M. Lewenstein, P. Balcou, M. Y. Ivanov, A. L'Huillier, and P. B. Corkum, "Theory of high-harmonic generation by low-frequency laser fields," Phys. Rev. A 49, 2117-2132 (1994).

36. M. Sabbar, S. Heuser, R. Boge, M. Lucchini, L. Gallmann, C. Cirelli, and U. Keller, "Combining attosecond XUV pulses with coincidence spectroscopy," Rev. Sci. Instrum. 85, 103113 (2014).

37. J. J. Moré, "The Levenberg-Marquardt algorithm: implementation and theory," in Numer. Anal., (Springer, 2006), pp. 105-116.

38. A. N. Grum-Grzhimailo, M. N. Khaerdinov, and K. Bartschat, "Effects of numerical approximations in the treatment of short-pulse strong-field ionization of atomic hydrogen," Phys. Rev. A 88, 055401 (2013).

39. N. Douguet, A. N. Grum-Grzhimailo, E. V. Gryzlova, E. I. Staroselskaya, J. Venzke, and K. Bartschat, "Photoelectron angular distributions in bichromatic atomic ionization induced by circularly polarized VUV femtosecond pulses," Phys. Rev. A 93, 033402 (2016).

40. X. M. Tong and C. D. Lin, "Empirical formula for static field ionization rates of atoms and molecules by lasers in the barrier-suppression regime," J. Phys. B 38, 2593-2600 (2005).

41. M. S. Pindzola, F. Robicheaux, S. D. Loch, J. C. Berengut, T. Topcu, J. Colgan, M. Foster, D. C. Griffin, C. P. Ballance, D. R. Schultz, T. Minami, N. R. Badnell, M. C. Witthoeft, D. R. Plante, D. M. Mitnik, J. A. Ludlow, and U. Kleiman, "The time-dependent close-coupling method for atomic and molecular collision processes," J. Phys. B 40, R39-R60 (2007).

42. T. N. Rescigno and C. W. McCurdy, "Numerical grid methods for quantum-mechanical scattering problems," Phys. Rev. A 62, 032706 (2000).

43. J. Feist, S. Nagele, R. Pazourek, E. Persson, B. I. Schneider, L. A. Collins, and J. Burgdörfer, "Nonsequential two-photon double ionization of helium," Phys. Rev. A 77, 043420 (2008).

44. S. Donsa, I. Brezinová, H. Ni, J. Feist, and J. Burgdörfer, "Polarization tagging of two-photon double ionization by elliptically polarized XUV pulses," Phys. Rev. A 99, 023413 (2019).

45. J. Vinbladh, J. M. Dahlström, and E. Lindroth, "Many-body calculations of two-photon, two-color matrix elements for attosecond delays," Phys. Rev. A 100, 043424 (2019).

46. U. Heinzmann and J. H. Dil, "Spinorbit-induced photoelectron spin polarization in angle-resolved photoemission from both atomic and condensed matter targets," J. Phys. Condens. Matter. 24, 173001 (2012).

47. U. Heinzmann, Attosecond Physics, Springer Series in Optical Sciences (Springer, 2013), Vol. 177.

48. M. Fanciulli, H. Volfová, S. Muff, J. Braun, H. Ebert, J. Minár, U. Heinzmann, and J. H. Dil, "Spin polarization and attosecond time delay in photoemission from spin degenerate states of solids," Phys. Rev. Lett. 118, 067402 (2017).

49. L. H. Haber, B. Doughty, and S. R. Leone, "Energy-dependent photoelectron angular distributions of two-color two-photon above threshold ionization of atomic helium," Phys. Rev. A 84, 013416 (2011).

50. M. Lucchini, A. Ludwig, T. Zimmermann, L. Kasmi, J. Herrmann, A Scrinzi, A. S. Landsman, L. Gallmann, and U. Keller, "Anisotropic emission in quantum-beat spectroscopy of helium excited states," Phys. Rev. A 91, 063406 (2015). 